\title{
Cost Decision Support in Product Design
}

\author{
A. Liebers \& H. J. J. Kals (1), Laboratory of Production and Design Engineering, Faculty of Mechanical Engineering, \\ University of Twente, The Netherlands \\ Received on January 7, 1997
}

\begin{abstract}
The constraints addressed in decision making during product design. process planning and producticn planning determine the admissibie solution space for the manufacture of products. The solution space determines largely the costs that are incurred in the production process. In order to be able to make economically sound decisions, costing data suppont must be integrated into the decision making processes. Regarding product design, the designer must be supplied with transparent costing data, that is ready for direct application. In this paper a functional architecture for costing data support during product design, as well as a corresponding data structure are presented.

Keywords: Cost Estimating, Design.
\end{abstract}

\section{Introduction}

Product design is a very important task regarding manufacturing costs. Apart from costs, two other performance criteria must be observed for every decision making process in manufacturing. They are the throughput time (or delivery date) and the (functional) product quality. Product design transiorms the functional requirements into a model of a physical object, capable of performing the required functions. Regarding costs, product design fixes up to $80 \%$ of the total product costs [6]. A part of these costs are necessary to obtain the required functionality. The other part of the costs, referred to as 'unnecessary' costs, must be avoided. Unnecessary costs result from an insufficient consideration of the objectives of the subsequent planning tasks during design. Furthermore, the inability to evaluate the cost consequences of design decisions leads to poor solutions from a cost point of view.

To overcome this problem, several methods can be used To be able to make cost-effective decisions, general guidelines should be applied in design [17]. Furthermore, cost models must lead to adequate cost estimation based on as little information as possible, like in function costing [7]. To consider subsequent manufacturing tasks during design, the 'Design For $X$ ' (DFX) [15] approach is being developed. DFX comprises various methods, like designfor-manufacturing-and-assembly [2] and design-for-lifecycle-costs [3]. For a reliable allocation of costs to products, traditional accounting methods have been turned up-side-down, by the introduction of activity based costing [9].

DFX is characterized by the fact that all the methods incorporated comprise design guidelines which only focus on one performance criterion. Instead of offering support during decision making, the design process is driven into a specific direction. For instance, design for assembly does not consider the aspect of the availability of resources. Problems can occur when a product is designed to suit a specific process and resource set, whereas the required resources are not available in the period in which production must take place. The use of alternative, less suited, resource sets increases the costs and/or the throughput times. Furthermore, the aforementioned cost models and general guidelines are only suited for a specific application area. Consequently, there is a need for an architecture to be developed, that specifies the support for the entire sequence of decision making processes with ready-for-use costing data. This publication focuses on the description of such a generic architecture with an emphasis on product design. Firstly, the situation encountered in manufacturing is discussed. Secondly, an architecture for solving the cost control problem is presented. Thirdly, the underlying data structure is discussed. Unless defined separately, in this publication the terminology of Chisholm [4] is adopted.

\section{Analysis of the costing support process}

The manufacturing cycle can be decomposed into activities that are related to the development of a plan, as well as activities that are related to the execution of that plan. The generation of a plan upon which production can be based involves a large number of decision making processes. Following a particular sequence in decision making processes must finally lead to a complete instruction set, required for the production. In this sequence of decision making, the freedom for every decision to be made is restricted by constraints imposed by preceding decisions. Furthermore, a decision making process limits the solution space for all subsequent decisions. The freedom in sequencing the decisions is limited and depends on the strategy of the regarded company. An information analysis is required upon which the selection of best solution for the sequence in decision making is based. Furthermore, there is a certain logical preference, limiting the number of alternative sequences. It may be obvious that costing support must be adapted to the needs of the decision making processes. 
Decision making can be related to various manufacturing tasks, like product design, resource engineering, process planning and production planning. For every decision making process, the three aforementioned performance criteria must be satisfied, to avoid unnecessary recursion. The product manufacturing cycle used to be regarded as a fixed sequence of tasks, in which every task had to be completed before the next task could be executed. The concept of concurrent engineering [14] suggests that the various tasks must partially be executed in parallel. Design no longer has to be completed before process planning is regarded, for instance. The decision making process is therefore characterised by small incremental steps.

The aforementioned view on decision making leads to the need to integrate the required design decisions [13], [16], with the other decision making processes in the company. Current developments in the field of DFX are attempts to solve this problem. Decision making in general is characterized by the fact that alternative solutions have to be generated and evaluated. Evaluations must regard all three performance criteria, to be able to compare the various alternatives and to select the most suitable alternative(s) for further development. Regarding costs, both qualitative and quantitative comparisons can be applied. The required level of detail, and therewith the method of comparison must be specified by the decision maker.

The costing support data is developed by some cost estimation process. Cost estimation determines the expected costs, using incomplete and possibly (partially) fictional data. In the case that quantitative costing data is generated, the cost estimate must include the expected cost value, together with a range indicating the best and the worst case. Furthermore, an estimate must include a specification of the assumptions upon which the estimate has been based.

\section{Design of a functional architecture for cost control}

The basic elements of cost control

Based on the analysis of the costing support process, a functional architecture is developed for cost control. Cost control consists of four major functions [11]. These functions are depicted in Figure 1. The first function is the cost estimation function (F1). The task of cost estimation is to calculate the costs for a production situation which is likely to be encountered. Cost estimation avails itself of cost models and cost-rates. A cost-driver is a product characteristic that has a noticeable influence on the costs of the product. Cost-drivers can be based on a quantity, or on an amount of time. The cost-driver is used to allocate costs to a 'cost-carrier', based on the characteristics of that cost-carrier. The relation between a cost-driver and a cost-carrier is frequently referred to as a 'cost-rate'. The costs to manufacture a product can be determined by identifying independent cost-carriers in a product, estimating their costs and accumulating these costs.

Cost-carriers can either be direct or indirect. Direct costcarriers are directly related to products. Examples of direct cost-carriers are for instance complete products or product elements. Indirect cost-carriers eventually have to be allocated to direct cost-carriers. Examples of indirect cost-carriers are for instance set-ups or resource sets.
Partially based on the generated cost estimate, a decision

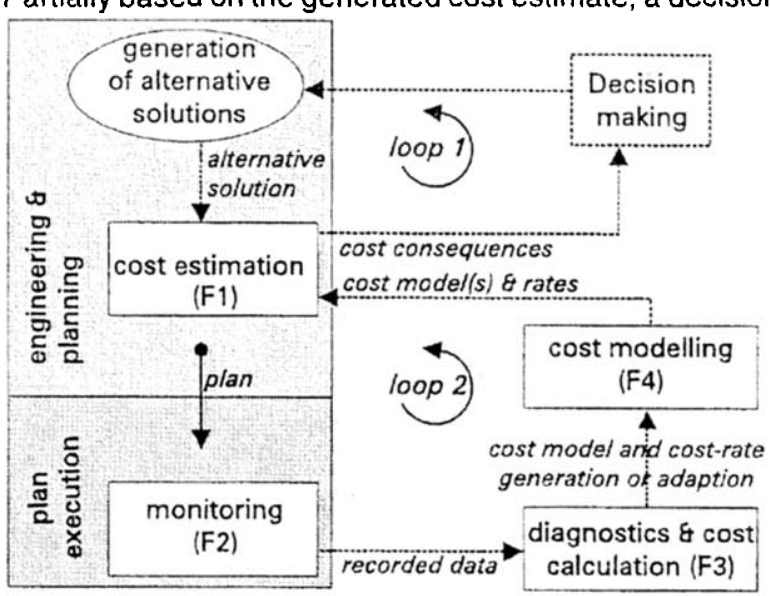

Figure 1:

A functional architecture for cost control

is made. The second function in cost control is the monitoring function (F2). Monitoring concerns the acquisition of data on the execution of the production process. Time can be recorded, as well as characteristics of the activities performed. Furthermore, disturbances can be monitored and recorded. Finally, resource performance data can be obtained, like average machine tool loads. The third function in cost control is the diagnostics and cost calculation function (F3). The data obtained during monitoring must be interpreted. From this data, conclusions can be drawn about the progress of orders. Using the actually encountered situation, also the actual costs can be calculated. The fourth function in cost control is the cost modelling function (F4). The data that have been interpreted by the diagnostic function are used to generate cost models, representing the relation between a cost-carrier and one or more cost-drivers. Data are analyzed to determine the influential characteristics of cost-carriers and to select the appropriate corresponding cost-drivers. To enable cost modelling, a feedback loop must exist. This loop is denoted with 'loop 2' in Figure 1. Furthermore, by comparing a cost estimate and the corresponding cost calculation, the quality of the assumptions made during estimation can be judged and, when needed, adapted for future use. For this purpose both cost estimation and calculation are based on the same cost models. Finally, by regarding the calculated product cost and feedback indicating the money flow that leaves the company, the correctness of the cost rates can be assessed. Apart from the four functions and the feedback, Figure 1 depicts the aforementioned nature of decision making ('loop 1').

\section{The anatomy of a costing support function}

As indicated, the task of cost estimation is the generation of costing support data. The anatomy of costing support is generic in nature, in that it is almost the same for every decision making process, including design decisions. Product design takes place early in the manufacturing cycle. At the moment of design, there is still little knowledge about the characteristics of the situation that will be encountered during production. The result is either a high degree of remaining uncertainty or much effort required to specity the expected situation in detail. In 
decision making, usually several alternative solutions can be generated that all represent a potential solution to the encountered problem. By specifying the situation that would result from the selection of a specific alternative, costs can be estimated for the regarded alternative. This aspect is depicted in Figure 1 by 'loop 1'. The cost implications of the decision can be obtained by estimating the costs for every alternative solution. By doing the same for the delivery time and the functional quality criteria, an adequate alternative solution can be selected. The three performance criteria need not be equally important. The relative value that is assigned to each of the performance criteria depends on the preference of the decision maker. This aspect is specified in a decision making strategy, together with the sequence of decision making and a history of constraints. The latter contains constraints that result from the functional requirements and previously performed decision making processes.

\section{A functional anchitecture for costing support in design}

The above finally results in the design of a functional architecture of the costing support function (in design). This architecture is represented in Figure 2 as an IDEF-O diagram [12]. In IDEF-O a function is depicted as a rectangle, with information flows coming from and leading to this function. From the top side, the 'costing data generation' function rectangle, is provided with control information. The control information comprises the initiation of the cost estimation process, together with the estimation requirements, like the accuracy. On the

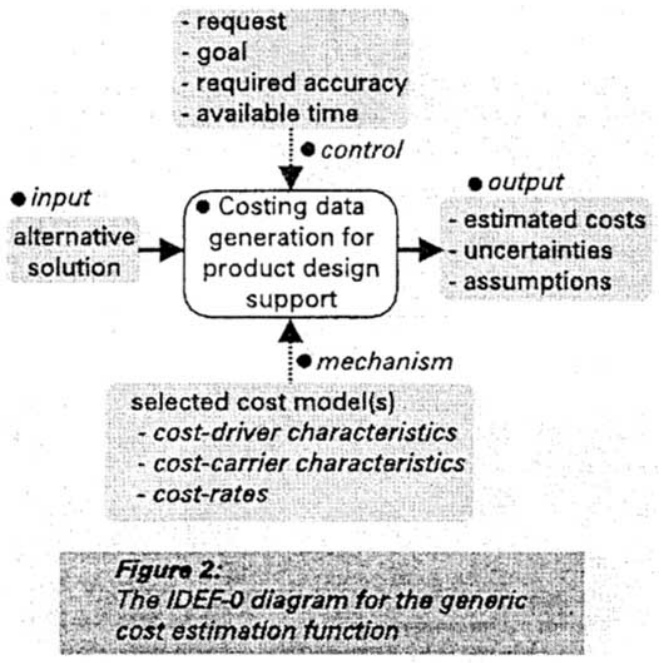

left-hand side, the input information is supplied. The input information describes the situation for which the costing support function is executed. This information includes a full description of the regarded alternative solution for which an estimate has been prepared. On the bottom side of the function box, the so called 'mechanism' is supplied. The mechanism represents the means with which the input can be transformed into the output. In costing support for design decisions, the mechanism consists of selected cost models that suit the specific situation, regarding the input and control information. To be able to select the suited cost models the mechanism must provide some 'filter'. A simple example of a cost function can be found in the case of aluminum extrusion, requiring the type and the length of the profile as input parameters. The type of profile indicates a cost factor that is multiplied with the length of the profile to obtain a cost estimate. The output of the function can be used to evaluate the cost consequences of a specific decision. Instead of an immediate evaluation, based on the available information, the accuracy needed in the cost estimate can require the generation of additional information. A more detailed development of the alternatives, enables a more detailed cost estimation, leading to a more refined evaluation of the alternatives. The potential level of detail that can be obtained depends on the importance of the specific decision and on the time available for generating the required information. Once a specific alternative has been selected, the additional detailed information can immediately be put to use.

Regarding the above, a major problem in evaluating the alternatives is the large number of decisions in the manufacturing process. For a high level decision, requiring a detailed evaluation, an explosion of the number of potential alternative situations can occur. To avoid this explosion of data, only a limited number of 'most promising alternative solutions' must be included in the evaluation. A fortunate side-effect regarding the risk of data explosion, is the fact that many decision making processes frequently recur for the company-specific manufacturing environment. Therefore, costs can be related to aggregated cost-carriers on the higher hierarchical levels in the product (assembly) structure, while satisfying the requirements for the level of detail. The hierarchical level on which the information is integrated into information units is defined as the level of aggregation'.

\section{Levels of aggregation}

As indicated before, costs can be allocated to costcarriers that are situated on various levels of aggregation. Complete products can for instance be used as costcarriers. A method in which products are used as costcarriers is 'parametric cost estimation'. It is also possible to relate costs to other physical elements in the product assembly structure. These physical elements include functional modules, assemblies and parts. When the part level does not provide cost information at the required level of detail, there is a need for more detailed planning (including process an production planning) to obtain adequate cost information. Features can be seen as an important link between design and process planning. Usually a limited set of features is applied. For this reason, costs can partially be allocated to features. Further detail can be obtained by regarding generic shapes, possibly in relation with individual operations that are required for the production of the regarded generic shape or a feature. The individual operations, including knowledge on the required resources, represent the most detailed input possible for cost estimation. A corresponding problem that must be kept in mind is the interdependency of the resource set used and the set of operations that is performed in one set-up. Usually, resources are allocated to a processing set-up for its entire duration.

To avoid the need for detailed planning information, similarity of cost-carriers on a high level of aggregation is preferred over similarity on some lower level. Regarding the latter there is a problem, since $100 \%$ similarity between the regarded cost-carrier and earlier encountered cost-carriers is very rare. To overcome this problem, classes of objects with certain characteristics are defined, including entities on the basis of which the degree of similarity can be determined. These classes are defined for each level of aggregation. The use of classes of objects can be seen as the aforementioned 'filter' with which appropriate cost models are identified [10]. This aspect is depicted in Figure 3a. The use of cost-carriers 


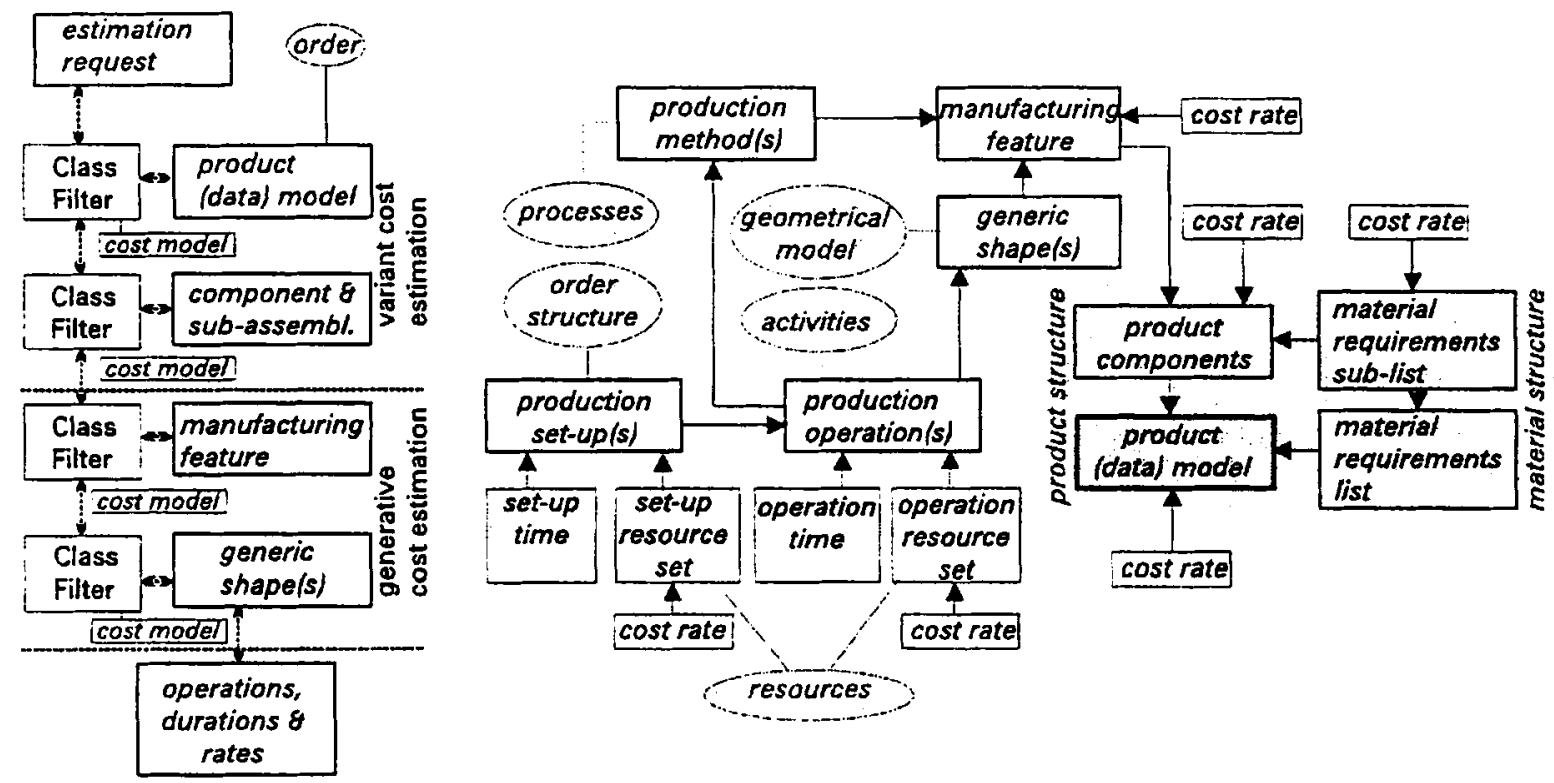

\author{
Figure 3a: \\ Levels of aggregation \\ and cost estimation
}

\author{
Figure 3b: \\ Simplified data model, using time and quantity related cost-carrlers. \\ based on the 'manufacturing information building block (1)
}

on high aggregation levels is usually referred to as variant-based cost estimation. The use of cost-carriers on the lower levels of aggregation, leading to much planning and recombination effort, is referred to as generative cost estimation (Figure 3a). The applicability of high level costcarriers depends mainly on the familiarity with the encountered situation.

\section{Data structure}

The functional architecture is directly related to a data structure upon which the required functionality can be built. The elements in the data structure correspond to the various cost-carriers and the related cost-drivers. Figure 3b depicts a data structure for costing support during product design. This model has been simplified for reasons of transparency. The links with the production knowledge, the production activities, the geometrical product model, the factory lay-out and the order structur $e$ are indicated by the shaded ovals.

\section{Example}

The use of cost information for decision support is illustrated by a simple example of a fictional product. Assuming two alternative embodiment designs, a cost estimate is required to determine the best alternative. The cost estimation process for one of the alternatives is indicated here. Various aggregation levels are addressed. To minimise the estimation effort, top-level physical entities are preferably used as cost-carriers. By determining the corresponding cost-functions and inserting the parameter values of the cost-drivers, the costs can be determined. The regarded alternative product design (Figure 4 ) is composed of two assemblies (a1 and a2) and of two parts ( $p 1$ and $p 2$ ). Part $p 1$ is subcontracted to another firm. Part $p 2$ is to be manufactured exclusively for the regarded order. Both assemblies consist of two parts (a 1p1, a1p2, a2p1 and a2p2). Assembly a1 is a standard functional module, consisting of two standard parts, assembled in a standard way. Assembly a2 is composed of two parts that are both manufactured in-house. Experience with $22 p 1$, resulting from a similarity search, has lead to the knowledge that the material costs make up almost the complete costs of the part. The other part (a2p2) is a relatively simple part belonging to a family of which many members have been manufactured before. The levels of aggregation encountered in this example include:

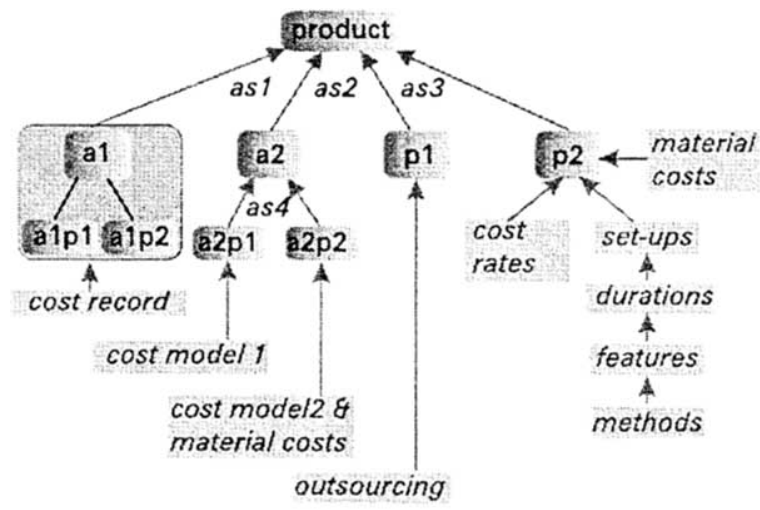

Figure 4:

An illustration of the cost estimation process

- product level

- assembly level

- part level

- set-up level

- operation level

The equations for the cost estimation process are depicted in Table 1. In this set of equations, $c_{x}$ represents the cost of entity $x$, indicating for instance the product, an assembly, a part, or an operation. Furthermore, $c_{\text {matorat }}$ represents the material cost, $c_{\text {mat.unit }}$ is the cost per unit of material and $c_{\text {production }}$ represents the total production cost 


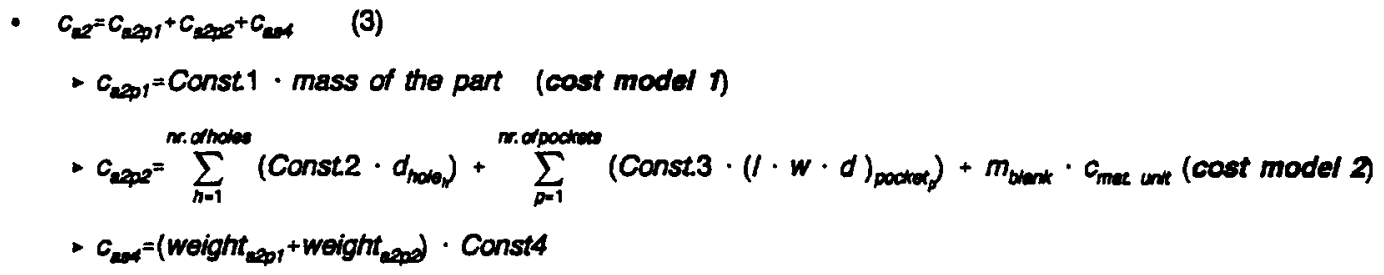

- $c_{p t}=$ value set by vendor (for 1 snr. purchasedkt0)

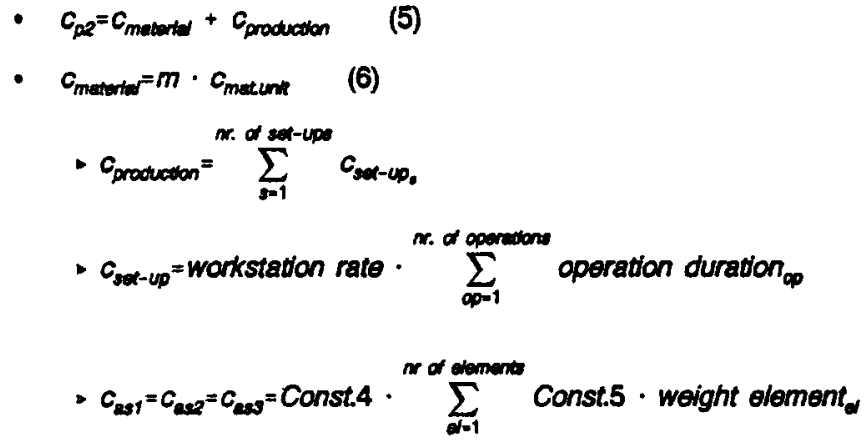

Table 1. The cost-functions for determining the product costs

of the regarded object. Finally, $m$ represents the number of material units, whereas $d_{\text {hole }}, l, w$ and $d$ respectively represent the diameter of a round hole and the length, width and depth of a pocket.

In general, for high level physical entities, the costs can be estimated by regarding the entity as a whole using historic cost information. The use of a template and the retrieval of a related cost record is the basis of the cost estimation process for the regarded physical entity. The detection of similarity and difference among various physical entities is discussed in [10] and [11]. Corrections have to be made to the retrieved cost record, to account for non-similarity between the template and the regarded physical entity. When no applicable template is available, the costs must be obtained by regarding the constituent physical entities on a lower level and the required assembly processes. The costs of the top level entity can be found by the use of a cost-function (equation 1 in Table 1). In this example, the cost-carrier 'product is initially subdivided into seven cost-carriers. The required cost-function is generic in nature, in that it includes the material costs and the production costs for the constituent elements, as well as the costs for the assembly process of the regarded physical entity.

To determine the costs of the constituent physical entities, cost estimation is again preferably based on templates. When no suitable templates can be found for an entity, the regarded entity is decomposed into constituent lower level entities. This process is continued until either for every assembly a template has been found, or until the lowest level entities are reached. The entities on the lowest level are the parts that constitute the product. For the individual parts a template can be used to obtain a cost estimate. When no template is available for a part, an estimate has to be produced using a cost-function. This estimate can be either a detailed or a rough cost estimate, depending on the relative importance of the regarded physical element and the required accuracy of the estimate. The generic function for cost estimating is based on a summation of the material costs and the production costs. By estimating the costs of the required blank, as well as the costs of all the required operations (including set-up operations, measurement operations, processing operations, etc.), the costs for the part can be found.

Cost-functions can be simplified to reduce the required estimation effort. This aspect is illustrated in Table 1 by cost models 1 and 2 . Another example would be the use of an equation including characteristics like the material type, the mass, the number of machined faces and the critical tolerances of the regarded part. By using this type of characteristics as cost-drivers, company specific information can be used for the purpose of cost estimation. The relation between company specific costdrivers and costs can be determined by applying techniques like regression analysis to production data. The development of cost-models lies beyond the scope of the present research, which is the determination of a generic architecture for cost control and cost estimation.

\section{Conclusions and recommendations}

Product design has a large influence on manufacturing costs. To be able to control these costs, the influence of alternative solutions on costs must be evaluated before an economically justified decision can be made. A generic functional architecture for cost estimation is presented. The functionality can be used as a decision support tool. The architecture is applicable for all engineering and planning related tasks. Therefore it can also contribute to an integration of these tasks. The effort required for the generation of costing support information, depends on the required level of detail and on the familiarity with the 
encountered situation.

Al this moment, the sub-tasks in product design, process planning and production planning are observed in more detail. The goal of this analysis is the identification of the required costing support information for each decision making process. The required support information must correspond with the required level of detail and the sequence in which the decision making processes are executed.

\section{References}

[1] Arentsen, A.L., 1995, A generic architecture for factory activity control, PhD thesis, Enschede.

[2] Boothroyd, G., 1994, Product design formanufacture and assembly, Computer aided design, volume 26 , no. 7, pp 505-520.

[3] Bras, B. \& Emblemsvàg, J., 1995, Activity-based costing and uncertainty in designing for the life cycle, Design for $X$ : Concurrent engineering imperatives. Huang, G.Q. (ed).

[4] Chisholm, A.W.J, 1990, Nomenclature and definitions for manufacturing systems, Annals of the CIRP. Vol.39/2, pp 735-742.

[5] Ehrlenspiel, K., 1985, Kostengünstig Konstruieren, Konstruktionsbücher, band 35, Berlin.

[6] Eversheim. W. 1990, Organisation in der Produktionstechnik, Band 2, VDI-Verlag, Düssidort.

[7] French, M.J., 1990, Function costing: a potential aid to designers, $J$. of engineering design, vol. 1, no. 1 .

[8] Hundal, M.S., 1993, Designing to costs, Concurrent engineering, Parsaei (ed.) \& Sullivan (ed.), Cambridge, pp 329-351.

[9] Kaplan, R.S., 1982, Advanced management accounting, Englewood Cliffs

[10] Liebers, A., Streppel, A.H., Schuttert, M.A., Kals, H.J.J., 1996, Pant classification for variant cost estimation, Proceedings of the SheMet '96 conference, pp 167-178, Enschede.

[11] Liebers, A. \& Kals, H.J.J., 1996, Integrated cost estimation for assembled products, Proceedings of the 28th CIRP international conference on Manufacturing Systems, pp 243-250, Johannesburg.

[12] Marca, D.A. \& McGowan, C.C., 1988, SADTे structured analysis and design technique, New York.

[13] Pahl, G. \& Beitz, W., 1996, Engineering design, a systematic approach, second edition, Berlin.

[14] Solhenius, G., 1992, Concurrent engineering, Annals of the CIRP vol. 41/2, pp 645-655.

[15] Storm, T., Tichem, M. \& Willemse, M.A., 1996, DFX, a tool for designers, Proceedings of the 28th CIRP international conference on Manufacturing Systems, pp 198-202, Johannesburg.

[16] Uilman, D.G., 1992, The mechanical design process, New York.

[17] VDI-Richtlinien nr. 2235, 1987, Wirtschaftliche Entscheidungen beim Konstruieren. 